\section{俩 Heighten Science \\ P U B L I C I T I O N S Corporation \\ ISSN \\ 2573-6264}

\title{
Kinesio taping in patients with shoulder impingement
}

\author{
Sorin P*, Ramirez FJ, Joly A, Patiño 0 and Terrasa S \\ Department of Physical Therapy, Hospital Italiano of Buenos Aires, Autonomous City of Buenos \\ Aires, Argentina
}

*Address for Correspondence: Pablo Sorin, Department of Physical Therapy, Hospital Italiano of Buenos Aires, Autonomous City of Buenos Aires, Argentina,

Email: pablo.sorin@hospitalitaliano.org.ar

Submitted: 08 May 2019

Approved: 21 May 2019

Published: 22 May 2019

Copyright: (c) 2019 Sorin P, et al. This is an open access article distributed under the Creative Commons Attribution License, which permits unrestricted use, distribution, and reproduction in any medium, provided the original work is properly cited

Keywords: Subacromial; Physical therapy; Acromiohumeral distance; Goniometer;

Ultrasound system

Check for updates

\section{Abstract}

\section{Study Design: Prospective Observational Study}

Background: Physical therapy is one of the primary treatment options for these patients, however, we were unable to identify previously published research that objectively assesses the impact of taping on joint range of motion or on pain levels in patients with SAIS. This gap in the literature motivated the present study.

Objectives: To describe the results of the application of neuromuscular taping in patients with subacromial impingement syndrome (SAIS), with respect to their pain levels, joint range of motion, and acromiohumeral distance, assessed via ultrasound

Methods: A prospective series of cases was evaluated at the Hospital Italiano de Buenos Aires between April and September of 2018. Twenty-four patients from age 25 to 65 were invited to participate ( 9 men and 15 women), all of whom had been diagnosed with rotator cuff tendinopathy, impingement syndrome, or bursitis with positive signs of impingement in the affected shoulder and anterior shoulder pain in flexion

Result: Significant changes in AHD (Figure 2), pain, and anterior flexion were registered after taping. Superficial dermatitis was detected in only two patients, but this did not affect the assessment.

Conclusion: In the results obtained by this study, it has been observed that the application of neuromuscular taping using the method described above significantly increases AHD, leading to short-term improvements in pain and joint range of motion.

\section{Introduction}

Shoulder pain is the second most common musculoskeletal disorder following low back pain [1], and a frequent affliction among workers and athletes. It has an incidence of nearly 30 new cases per 1000 individuals per year, and among those aged 18 and 44 its prevalence is almost $22 \%$ [2].

Subacromial impingement syndrome (hereinafter SAIS) is one of the most frequent causes of shoulder pain and functional deterioration $[3,4]$. Impingement of the tendons of the rotator cuff, biceps, and soft tissue between the coracoacromial arch and the humeral head causes edema, inflammation, and shoulder pain [5]. There are structural and functional factors that lead to subacromial crowding, and subsequently SAIS. Weakness of scapular muscles and those of the rotator cuff, a type III acromion, and soft tissue retractions are some of such factors.

Conservative treatment of SAIS includes a wide variety of therapies that aim to diminish pain and improve joint mobility. Treatments include: exercise protocols for muscular strengthening and stretching [6], corticosteroid injections, cryotherapy, acupuncture, thermotherapy, manual therapy [7], prolotherapy, manipulations, 
ultrasound and transcutaneous electrical nerve stimulation (TENS) [8,9], extracorporeal shock wave therapy, joint mobilization techniques, magnetic fields, and various alternative therapies [10]. Nonetheless, there is still a lack of solid scientific evidence to support the use of these treatments, and it should be noted that when so many treatment options exist for a determined pathology, it is reasonable to think that none shows clear evidence of superiority over the rest.

Physical therapy is one of the primary treatment options for these patients, and in recent years new techniques have been developed such as neuromuscular taping commonly known simply as "taping" - which basically consists of attaching an adhesive and elastic band on the affected zone with the objective of regulating biological processes such as pain, inflammation, muscle activation, and circulation [11-13].

In patients with SAIS, taping seeks to reposition the shoulder complex in order to augment the subacromial space. Some authors [14], have documented improvements in pain and immobility in patients with rotator cuff tendinitis and shoulder impingement.

Nonetheless, the effectiveness of taping and its influence on results are not conclusive, given that in the majority of cases it was combined with a supervised shoulder exercise program. It is therefore difficult to determine the fraction of improvement attributable to each of the therapeutic interventions $[15,16]$.

The height of the subacromial space is central for normal shoulder functioning; this can be assessed by measuring the acromiohumeral distance (AHD), which is the distance between the acromion and the apex of the humeral head. When carried out by a trained professional, scapular taping can augment the AHD and facilitate rotator cuff tendon gliding in shoulder movements [17]. However, we were unable to identify previously published research that objectively assesses the impact of taping on joint range of motion or on pain levels in patients with SAIS. This gap in the literature motivated the present study.

\section{Objective}

To describe the results of the application of neuromuscular taping in patients with subacromial impingement syndrome (SAIS), with respect to their pain levels, joint range of motion, and acromiohumeral distance, assessed via ultrasound.

\section{Methods}

A prospective series of cases was evaluated at the Hospital Italiano de Buenos Aires between April and September of 2018. Twenty-four patients from age 25 to 65 were invited to participate ( 9 men and 15 women), all of whom had been diagnosed with rotator cuff tendinopathy, impingement syndrome, or bursitis with positive signs of impingement in the affected shoulder and anterior shoulder pain in flexion (these were the inclusion criteria). The exclusion criteria for participation in this study included having underwent previous shoulder surgery, rotator cuff calcifications, cervical radiculopathy, fractures, luxations, adhesive capsulitis, and neurological disorders with effects on the upper limb.

The research protocol was approved by the Ethics and Research Protocol Committee at the Hospital Italiano de Buenos Aires, and all participants provided written informed consent.

Description of procedures and measurements.

Three assessments were carried out on each of the 24 patients - one to establish the base line, one after the application of the therapeutic taping, and another three days after application. All relevant variables were assessed in each instance.

Pain was assessed using a visual analog scale (VAS). 
Joint range of motion was measured using a Prohab Mod U310 goniometer, using reference values of $0-150^{\circ} / 170^{\circ}$ for anterior flexion, $0-160^{\circ} / 180^{\circ}$ for abduction, $0-70^{\circ}$ for inward rotation, and $0-70^{\circ}$ for outward rotation (values defined by the $\mathrm{AO}$ Foundation - the Association for the Study of Internal Fixation). A cromiohumeral distance (AHD) measurement was carried out on all patients by the same technician. The ultrasound system used was a Philips HD-15 (Washington, USA) with a 12-5 MHz and 15-7 MHz linear transducer, depending on the patient's physical state. The patient was seated on the examining table with their upper limb in a neutral position and the transducer was placed in the coronal plane with its medial margin over the lateral edge of the acromion, such that the proximal extremity of the humerus and the acromion were visible in the same image. The AHD was measured vertically from the inferior border of the acromion to the superior border of the humeral head before and after taping.

As previously mentioned, therapeutic taping was applied immediately after the first ultrasound. Two strips of tape were applied, the first from the inferior scapular angle to the inferior surface of the midclavicular line, and the second from the inferior scapular angle to the inner surface of the bicipital groove. Both strips of tape were applied at $90 \%$ to $100 \%$ of their tension (joint correction technique) (Figure 1).

\section{Results}

Twenty-four patients were examined, with a median age of 50 years (15 were women, or $62.5 \%$ ) and a median pain evolution of four weeks. Their base line characteristics are described in the following table 1.

As the results in table 2 show, after application of therapeutic taping, a moderate augmentation of $0.65 \mathrm{~mm}(0.45$ to $1 ; \mathrm{p}<0.0001)$ was observed in the subacromial space, which was accompanied by a marked improvement in pain: $-3(-3$ to $-2 ; \mathrm{p}<0.0001)$ and an increase in range of motion for anterior flexion of the shoulder (17.5 (12.5 to 20) with $\mathrm{p}<0.0001$ ).

Significant changes in AHD (Figure 2), pain, and anterior flexion were registered after taping.

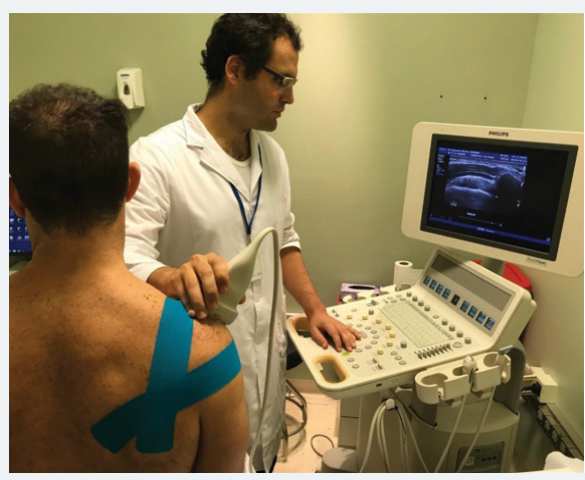

Figure 1: Ultrasound after application of taping.

Table1: Base line characteristics of 24 patients (15 women, $62.5 \%$ ) with subacromial impingement syndrome treated with therapeutic taping.

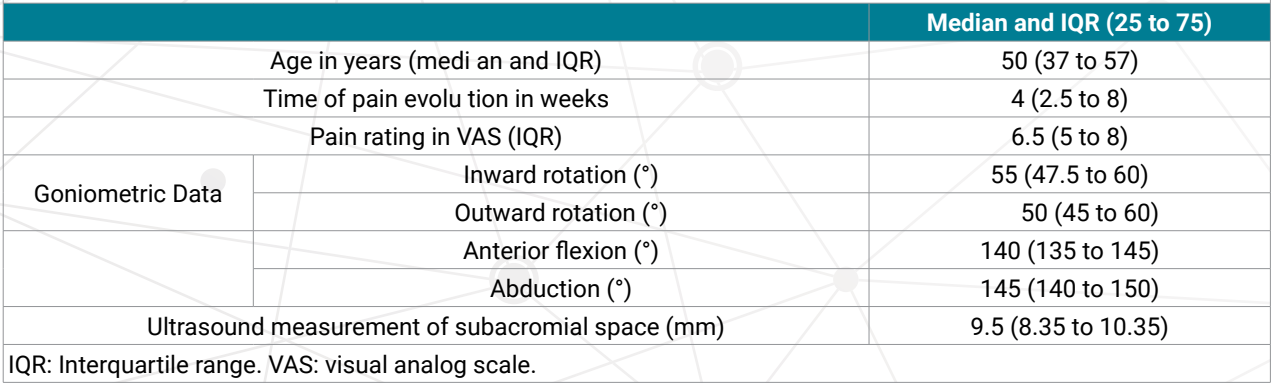




\begin{tabular}{|c|c|c|c|}
\hline & & $\begin{array}{l}\text { Change after the application } \\
\text { of taping. } \\
\text { Median and IQR ( } 25 \text { to } 75)\end{array}$ & $\mathrm{p}$ \\
\hline & Pain rating in VAS (IQR) ${ }^{1}$ & $-3(-3$ to -2$)$ & $<0.0001$ \\
\hline \multirow{4}{*}{ Goniometric Data } & Inward rotation $\left(^{\circ}\right)$ & $0(0$ to 5$)$ & 0.0362 \\
\hline & Outward rotation $\left(^{\circ}\right)$ & $0(0$ to 7.5$)$ & $<0.0010$ \\
\hline & Anterior flexion $\left({ }^{\circ}\right)$ & 17.5 (12.5 to 20$)$ & $<0.0001$ \\
\hline & Abduction $\left({ }^{\circ}\right)$ & $5(0$ to 10$)$ & $<0.0004$ \\
\hline \multicolumn{2}{|c|}{ Ultrasound measurement of sub acromial space $(\mathrm{mm})^{2}$} & $0.65(0.45$ to 1$)$ & $<0.0001$ \\
\hline
\end{tabular}

${ }^{1}$ Three days after the application of therapeutic tape. ${ }^{2}$ Immediately after the application of therapeutic tape. IQR: Interquartile range. VAS: visual analog scale.

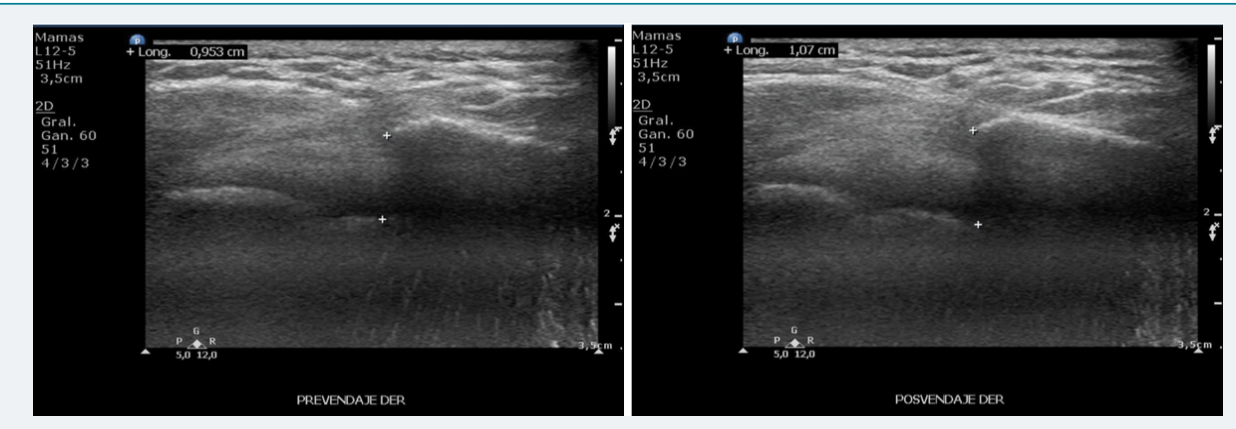

Figure 2: Ultrasound pre-and post-taping, which demonstrates change in AHD.

Superficial dermatitis was detected in only two patients, but this did not affect the assessment.

\section{Discussion}

We have presented a series of patients with subacromial impingement syndrome who have been closely monitored, and have observed an increase in AHD after the application of Kinesio Tape, which was accompanied by a clinically relevant improvement in pain and joint range of movement (particularly anterior flexion). These changes were maintained three days after the application of therapeutic tape.

We believe that a possible change in the mechanism of joint repositioning aided by the application of the Kinesio Taping method was responsible for the benefits brought about in patients with shoulder pain.

Regarding the limitations of our study, we should point out the absence of a control group and short monitoring period. Future studies that include a control group (one that is not treated with this method) and random assignment of treatment, as well as longer monitoring times, will allow for further assessment of the effectiveness of Kinesio Taping in patients with subacromial impingement syndrome.

\section{Especial thanks}

Physical Therapy Department - Hospital Italiano de Buenos Aires - Argentina

Diagnostic Imaging Department - Hospital Italiano de Buenos Aires - Argentina

\section{Conclusion}

In the results obtained by this study, it has been observed that the application of neuromuscular taping using the method described above significantly increases AHD, leading to short-term improvements in pain and joint range of motion.

\section{References}

1. Green S, Buchbinder R, Hetrick S. Physiotherapy interventions for shoulder pain. Cochrane Database Syst Rev. 2003; CD004258. Ref.: https://tinyurl.com/yyf3boyz 
2. Greeving K, Dorrestijn O, Winters JC, Groenhof F, van der Meer K, et al. Incidence, prevalence and consultation rates of shoulder complaints in general practice. Scand J Rheumatol. 2012; 41:150155. Ref.: https://tinyurl.com/y5o2gmkg

3. Van der Windt DA, Koes BW, Boeke AJ, Devillé W, De Jong BA, et al. Shoulder disorders in general practice: prognostics indicators of outcomes. Br J Gen Prac. 1996; 46: 519-523. Ref.: https://tinyurl.com/y4764uk2

4. Michener LA, Walsworth MK, Burnet EN. Effectiveness of rehabilitation for patients with subacromial impingement syndrome: a systematic review. J Hand Ther. 2004; 17: 152-164. Ref.: https://tinyurl.com/y427zvsf

5. Neer CS. Impingement lesions. Clin Orthop Relat Res. 1983; 70-77. Ref.: https://tinyurl.com/y5hv6bnc

6. Greeving K, Dorrestijn O, Winters JC, Groenhof F, van der Meer K, et al. Incidence, prevalence and consultation rates of shoulder complaints in general practice. Scand J Rheumatol. 2012; 41: 150155. Ref.: https://tinyurl.com/y5o2gmkg

7. Kampar A, Irrgang JJ, Whitney SL. Nonoperative management of secondary shoulder impingement syndrome. J Orthop Sports Phys Ther. 1993; 17: 212-224. Ref.: https://tinyurl.com/yyghr7lg

8. Senbursa G, Baltaci G, Atay A. Comparision of conservative treatment with and without manual physical therapy for patients with shoulder impingement síndrome: a prospective, randomized clinical trial. Knee Surg Sports Traumatol Arthrosc. 2007; 15: 915-921. Ref.: https://tinyurl.com/y63cvhco

9. Celik D, Atalar AC, Sahinkaya S, Demirhan M. The value of intermittent ultrasound treatment in subacromial impingement síndrome. Acta Orthop Traumatol Turc. 2009; 43: 243-247. Ref.: https://tinyurl.com/y4hzuvy4

10. Hsu CJ, Wang DY, Tseng KF, Fong YC, Hsu HC, et al. Extracorporeal shock wave therapy for calcifying tendinitis of the shoulder. J Shoulder Elbow Surg. 2008; 17: 55-59. Ref.: https://tinyurl.com/y4j8I5fy

11. Selkowitz DM, Chaney C, Stuckey SJ, Vlad G. The effect of scapular taping on the surface electromyographic signal amplitude of shoulder girdle muscles during upper extremity elevation in individuals with suspected shoulder impingement syndrome. J Orthop Sports Phys Ther 2007; 37: 694-702. Ref.: https://tinyurl.com/y59ajkqg

12. Morrisey D. Propioceptive shoulder taping. J Bodyw Mov Ther. 2000; 4: 189-194. Ref.: https://tinyurl.com/y2a4ykme

13. Smith $M$, Sparkes V, Busse M Enright S. Upper and lower trapezius muscle activity in subjects with subacromial impingement symptoms: is there imbalance and can taping change it? Phys Ther Sport. 2009; 10: 45-50. Ref.: https://tinyurl.com/y4atxyfe

14. Kase K, Wallis J, Kase T. Clinical therapeutical applications of the kinesio taping method. Tokyo, Japan. Kenikai Co Ltd. 2003. Ref.: https://tinyurl.com/yyfdt4bx

15. Thelen M, Dauber J, Stoneman P. The clinical efficacy of kinesio tape for shoulder pain: a randomized double blind clinical trail. J Orthop Sports Phys Ther. 2008; 38: 389-395. Ref.: https://tinyurl.com/y2w68jcq

16. Desmeules F, Minville L, Riederer B, Cote $\mathrm{CH}$, Fremont P. Acromio-humeral distance measured by ultrasonography and its association with the outcome of rehabilitation for shoulder impingement syndrome. Clin J Sport Med. 2004; 14: 197-205. Ref.: https://tinyurl.com/y4kfkzm6

17. Matsuki K, Matsuki KO, Yamaguchi S, et al. Dynamic in vivo glenohumeral kinematics during scapular plane abduction in healthy shoulders. J Orthop Sports Phys Ther. 2012; 42: 96-104. Ref.: https://tinyurl.com/yyynkgcx 